Marc-André Bédard Michel Panisset

\section{ADRESSES}

M.A. Bédard : chercheur boursier (FRSO) et professeur (UQAM). Service de neurologie, hôpital Notre-Dame, 1560, rue Sherbrooke Est, Montréal, Québec, H2L 4M1, Canada. M. Panisset : neurologue et professeur, université McGill. Centre McGill d'étude sur le vieillissement, hôpital Douglas, 6825, boulevard Lasalle, Verdun, Québec, H4H 1R3, Canada.

$\mathrm{m} / \mathrm{s} n^{\circ} \| 1$, vol. 11, novembre 95

\title{
Neurochimie des troubles cognitifs dans la maladie de Parkinson
}

Au cours des vingt dernières années, on a reconnu la fréquence, la nature et la physiopathologie des troubles cognitifs dans la maladie de Parkinson (MP). Certains syndromes y sont plus fréquents que dans la population générale, comme la maladie d'Alzheimer ou la maladie des corps de Lewy diffus. Toutefois, un tableau caractéristique de détérioration cognitive, connu sous le terme de syndrome souscortico-frontal (SSCF), semble le mieux décrire les troubles cognitifs de la majorité des patients atteints d'une MP. Le SSCF serait la conséquence d'une désafférentation du cortex frontal ou, d'un point de vue fonctionnel, de la désactivation de programmes cognitifs demeurés intacts. On ne connaît que partiellement la biochimie des voies sous-cortico-frontales et des programmes cognitifs qu'elle sous-tend dans le SSCF. Bien que la plupart des études aient mis l'accent sur les lésions dopaminergiques, l'exploration des systèmes cholinergiques, seuls ou dans leurs interactions avec les lésions dopaminergiques, s'avère beaucoup plus féconde.

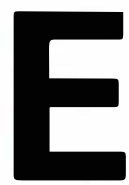
n 1817, James Parkinson décrivait la maladie qui porte aujourd'hui son nom comme l'association d'un tremblement, d'une bradykinésie et d'une rigidité, "en l'absence de toute altération des sens et de l'intellect" [1]. Dès lors, la maladie de Parkinson (MP) était considérée comme une affection essentiellement motrice. Il faut dire qu'à cette époque, l'état intellectuel pouvait difficilement être apprécié chez ces patients qui devenaient profondément akinétiques et dysarthriques. La coexistence de "troubles mentaux" ne fut affirmée que quarante-cinqans plus tard par Charcot et Vulpian [2]. Aujourd'hui, si les troubles cognitifs des parkinsoniens paraissent plus évidents, c'est sans doute parce que ces patients vivent plus longtemps et que la correction, au moins partielle, de leurs troubles moteurs rend plus facile l'évaluation de leur fonctionnement intellectuel. Toutefois, beaucoup d'incertitude demeure quant à la nature exacte, la sévérité ou même la prévalence des troubles cognitifs dans la MP. A titre d'exemple, la présence d'une démence dans la MP a été estimée à des proportions variant 


\section{RÉFÉRENCES}

1. Parkinson J. An assay on the shaking palsy. London, Sherwood, Nelly and Jones, 1817. (Réimpression dans Arch Neurol 1922, 7 : 682-710).

2. Charcot JM, Vulpian A. De la paralysie agitante. Gaz Hebd Med Chir 1861 ; 8: 765-7.

3. Rajput AH. Prevalence of dementia in Parkinson's disease. In: Huber SJ, Cummings JL, eds. Parkinson's disease: neurobehavioral aspects. Oxford: Oxford University Press, 1992: 119-31.

4. Ross GW, Mahler ME, Cummings JL. The dementia syndromes of Parkinson's disease cortical and subcortical features. In: Huber SJ, Cummings JL, eds. Parkinson's disease: neurobehavioral aspects. Oxford: Oxford University Press, 1992: 132-48.

5. Quinn N, Critchley P, Marsden CD. Young onset Parkinson's disease. Mour Dis $1987 ; 2: 73-91$.

6. Dubois B, Pillon B, Sternic N, Lhermitte F, Agid Y. Age-induced cognitive disturbances in Parkinson's disease. Neurology $1990 ; 40: 38-41$.

7. Chang Chui H, Perlmutter LS. Pathological correlates of dementia in Parkinson's disease. In : Huber SJ, Cummings JL, eds. Parkinson's disease: neurobehavioral aspects. Oxford: Oxford University Press, 1992: 16477.

8. Perry EK, Curtis M, Dick DJ, Candy JM, Atack JR, Bloxham CA, Blessed G, Fairbairn A, Tomlinson B, Perry RH. Cholinergic correlates of cognitive impairment in Parkinson's disease: comparaisons with Alzheimer disease. J Neurol Neurosurg Psychiatr 1985; 48 : 413-21.

9. Kosaka K. Dementia and neuropathology in Lewy Body Diseases. In : Narabayashi H, Nagatsu T, Yanagisawa N, Mizuno Y, eds. Parkinson's disease: from basic research to treatment. Advances in neurology, vol. 60. New York: Raven Press, 1993: 456-3.

10. Kusuhara S, Yoshimura M. Clinical and neuropathological aspects of Diffuse Lewy Body Disease. In: Narabayashi H, Nagatsu T, Yanagisawa N, Mizuno Y, eds. Parkinson's disease: from basic research to treatment. Advances in neurology, vol. 60. New York: Raven Press, 1993: 4649.

11. Cummings JL. Subcortical dementia. Oxford: Oxford University Press, 1990.

12. Sagar HJ, Sullivan EV, Gabrielli JDE, Corkin S, Growdon JH. Temporal ordering and short-term memory deficits in Parkinson's disease. Brain 1988; 111 : 525-39.

13. Canavan AGM, Passingham RE, Marsden CD, Quinn N, Wyke M, Polkay CE. The performance on learning tasks of patients in early stages of Parkinson's disease. Neuro- de $8 \%$ à $93 \%$ [3] selon les études. Ces écarts extrêmes s'expliquent en grande partie par le manque de consensus sur la définition de la démence dans la MP, car la symptomatologie des troubles cognitifs varie considérablement dans cette population.

\section{La démence dans la MP}

La plupart des auteurs reconnaissent aujourd'hui, selon leur état neuropsychologique, trois sous-populations de patients parkinsoniens: (1) un groupe sans atteinte cognitive; (2) un groupe avec démence de type Alzheimer associée; et (3) un groupe avec déficits cognitifs caractéristiques, reconnus sous le terme de syndrome sous-cortico-frontal (SSCF), qui rappelle les déficits associés aux lésions du cortex frontal [4]. Les malades qui développent une MP précocement, c'est-à-dire avant l'âge de 40 ans (MP juvénile), appartiennent en majorité au premier groupe et restent souvent sans déficits cognitifs notables, même après plusieurs années d'évolution de la maladie [5]. Les lésions neuropathologiques de ces patients sont habituellement bien circonscrites et limitées à la substance noire pars compacta. A l'opposé, les patients qui développent une MP tardive, c'est-à-dire après l'âge de 65 ans, présentent souvent une détérioration cognitive sévère, proportionnelle à la durée d'évolution de la maladie [6]. Cette forme de démence évolue rapidement et se présente, sur le plan clinique, comme une démence de type Alzheimer. Les études post-mortem chez ces patients [7] révèlent d'ailleurs que le cortex cérébral peut contenir un nombre important de plaques séniles et d'enchevêtrements neurofibrillaires, deux stigmates neuropathologiques caractéristiques de la maladie d'Alzheimer (MA). Pour la plupart des auteurs [7], la seule présence de ces éléments neuropathologiques en quantité anormale n'indique pas nécessairement la présence d'une MA concomitante à la MP. Il semble en effet que les éléments constitutifs des plaques séniles seraient différents dans ces deux populations; ceux retrouvés dans la MA contiendraient des formations neuritiques proéminentes, alors que ceux associées à la MP ou au vieillis- sement normal seraient sans neurites [7].

C'est sur le plan neurochimique qu'on a rapporté les plus grandes similitudes entre la MA et la MP. Mentionnons, notamment, une réduction des concentrations corticales de somatostatine, de même qu'une atteinte des fibres cholinergiques innominato-corticales et septo-hippocampiques $[8,9]$. Le degré d'atteinte de ces fibres cholinergiques est d'ailleurs plus grand chez les parkinsoniens déments que chez les parkinsoniens non déments, bien que, chez ces derniers, il n'y ait pas nécessairement de corrélation entre l'ampleur des dégénérescences et la sévérité des déficits cognitifs [9].

D'autres étiologies peuvent être mises en cause dans l'apparition d'une démence chez les sujets atteints d'une MP. Ainsi, on a montré chez certains parkinsoniens déments que le cortex et le diencéphale pouvaient contenir un nombre élevé de corps de Lewy (maladie des corps de Lewy diffus; MCLD) $[9,10]$; ce sont des stigmates caractéristiques de la MP, habituellement localisés dans la substance noire mais rarement observés au niveau du cortex. Chez ces malades, le syndrome démentiel précède habituellement le syndrome akinéto-rigide, bien que l'apparition inverse ou l'évolution simultanée des deux syndromes aient été aussi rapportées [9]. Toutefois, une controverse existe concernant le rôle des corps de Lewy corticaux depuis qu'on a démontré l'existence de MCLD sans démence associée [10]. Par ailleurs, un nombre anormalement élevé de plaques séniles et d'enchevêtrements neurofibrillaires est rapporté dans la plupart des cas de MCLD [10], ce qui suggère la nécessité d'une MA concomitante à la MCLD ou à la MP pour expliquer le syndrome démentiel de ces patients.

Le type de déficit cognitif retrouvé dans les démences avec atteintes corticales telles que la MA ou la MCLD implique toujours les fonctions instrumentales, c'est-à-dire le langage, les fonctions perceptivo-visuelles et la production de gestes symboliques ou complexes. On note également des troubles de la mémoire caractérisés principalement par un "oubli à mesure». Toutefois, la sévérité des déficits dans ces différentes sphères 


\begin{tabular}{|lll|}
\hline \multicolumn{2}{|c|}{ Tableau I } \\
COMPARAISON DES PROFILS CLINIQUES ENTRE \\
LA DEMENCE CORTICALE (OU DEMENCE DE TYPE ALZHEIMER) \\
ET LE SYNDROME SOUS-CORTICO-FRONTAL \\
(OU DEMENCE SOUS-CORTICALE)
\end{tabular}

Tableau II

\section{CRITĖRES DIAGNOSTIQUES DE DÉMENCE SELON LE DSM-IV}

\section{Troubles de la mémoire}

2. Un des critères suivants:
a. Troubles d'abstraction
b. Troubles du jugement
c. Troubles du langage (aphasie)
d. Troubles du geste (apraxie)
e. Troubles perceptuels (agnosie)
f. Troubles constructifs
g. Changements de la personnalité

3. L'atteinte de 1 et 2 affecte le fonctionnement social ou professionnel

4. État de conscience et de vigilance normaux

intellectuelles est très variable d'un patient à l'autre, d'où une certaine hétérogénéité des tableaux cliniques. Il est néanmoins possible de distinguer ces formes de détérioration cognitive, que l'on pourrait qualifier de démence corticale (DC) ou démence de type Alzheimer, par rapport à un autre tableau clinique de détérioration cognitive caractéristique appelé "démence sous-corticale" ou "syndrome sous-cortico-frontal " (SSCF), présent chez la plupart tiel au diagnostic de démence selon le ISM-IV* (Tableau II). Pour cette raison, il est préférable d'utiliser le terme de SSCF, afin d'éviter toute confusion possible avec la démence de type Alzheimer, parfois présente dans la MP.

\section{Le syndrome sous-cortico-frontal de la MP}

Le terme de SSCF est souvent utilisé pour mieux caractériser la nature des troubles intellectuels spécifiquement associés à une dysfonction des noyaux gris centraux. Le syndrome reste cependant non spécifique et peut être observé dans plusieurs affections sous-corticales [11], telles que les lésions thalamiques, les atteintes du troisième ventricule, l'hydrocéphalie normotensive, la sclérose en plaques, ou encore dans les maladies plus généralisées telles que le syndrome immunodéficitaire acquis, les maladies pulmonaires obstructives chroniques ou la dépression. Sur le plan clinique, le SSCF s'oppose à la démence de type Alzheimer par l'absence de troubles instrumentaux (troubles du langage, de la perception ou de l'élaboration des gestes), et par la présence de déficits cognitifs qui sont habituellement rencontrés lors de lésions corticales frontales (Tableau I), c'est-à-dire une inertie ou un ralentissement intellectuel (bradyphrénie), des indices de persévération $^{* *}$ (inflexibilité mentale et comportementale), des difficultés de planification et d'organisation dans les stratégies de travail et, enfin, des troubles de la mémoire. La nature des troubles de la mémoire dans le SSCF est également différente de celle rencontrée dans la démence de type Alzheimer. Par exemple, certains auteurs [12] ont montré la difficulté des parkinsoniens à se souvenir de l'ordre séquentiel des événements, sans oubli cependant des événements proprement dits. Cela peut être mis en évidence notamment par

\footnotetext{
* DSM-IV : diagnostic and statistical manual for mental disorders, $4^{\mathrm{c}}$ ed.

** Terme général indiquant le maintien d'une attitude, la répétition d'un geste ou de mots.
} 


\section{RÉFÉRENCES}

14. Pillon B, Deweer B, Agid Y, Dubois B. Explicit memory in Alzheimer's, Huntington's, and Parkinson's diseases. Arch Neurol $1993 ; 50: 3749$.

15. Boller F, Passafiume D, Keefe NC, Rogers K, Morrow L, Kim Y. Visuospatial impairments in Parkinson's disease. Arch Neurol $1984 ; 41$ : 485-90.

16. Brown RG, Marsden CD. Visuospatial function in Parkinson's disease. Brain 1986; $109: 287-1002$.

17. Brown RG, Marsden CD. Cognitive function in Parkinson's disease: from description to theory. Trends Neurosci $1990 ; 13$ : 21-9.

18. Brown RG, Marsden CD. Internal versus external cues and the control of attention in Parkinson's disease. Brain 1988; 111: $323-45$

19. Sharpe MH. Distractibility in early Parkinson's disease. Cortex 1990; $26: 239-46$.

20. Malapanis C, Pillon B, Dubois B, Agid Y. Impaired simultaneous cognitive task performance in Parkinson's disease: a dopamine-related function. Neurology 1994; 44: 319-26.

21. Panisset M, Simard M, Boller F, Cesaro $P$, Degos JD. Central executive system deficit in Parkinson's disease. In : Amos D, Korczyn, eds. Dementia in Parkinson's disease. Bologna: Monduzzi Editore, 1994 : 249-52.

22. Sawaguchi T, Goldman-Rakic P. D1 dopamine receptors in prefrontal cortex: involvement in working memory. Science $1991 ; 251: 947-50$.

23. Huber SJ, Glatt SL. Neuroimaging correlates of dementia in Parkinson's disease. In : Huber SJ, Cummings JL, eds. Parkinson's disease: neurobehavioral asperts. Oxford: Oxford University Press, 1992: 149-63.

24. Wallesch CW, Karnath HO, Zimmerman $P$. Is there a frontal lobe dysfunction in Parkinson's disease? A comparison of the effects of Parkinson's disease and circumscribed frontal lobe lesions in a maze-learning task. In: Vallar G, Cappa SF, Claus-W. Wallesch, eds. Neuropsychological disorders associated with subcortical lesions. Oxford: Oxford University Press, 1992: 227-41.

25. Taylor AE, Saint-Cyr JA, Lang AE. Frontal lobe dysfunction in Parkinson's disease. Brain 1986; 109 : 845-83.

26. Stacy M, Jankovick J. Clinical and neurobiological aspect of Parkinson's disease In : Huber SJ, Cummings JL, eds. Parkinson's disease: neurobehavioral aspects. Oxford des tests de discrimination chronologique. En revanche, la simple répétition d'une série de chiffres ou d'une séquence spatiale ne pose aucun problème à ces patients [13]. Cela suggère donc un déficit, non pas dans toutes les tâches impliquant un ordre temporel de l'information, mais seulement dans celles pour lesquelles le sujet doit effectuer une organisation active de cet ordre temporel. Cette difficulté d'organisation de l'information permet d'ailleurs d'expliquer l'ensemble des troubles de mémoire ou d'apprentissage propres au SSCF. En effet, ces patients éprouvent des difficultés dans des conditions de rappel par évocation libre, alors que les souvenirs paraissent bien préservés si le rappel se fait par reconnaissance ou à l'aide d'indices fournis aux patients [14]. Ainsi, à l'inverse de la démence de type Alzheimer, les patients avec un SSCF ne font pas montre d'oubli de l'information, mais plutôt d'une difficulté à établir des stratégies de rappel efficaces pour retrouver une information mnésique préservée.

Une telle perturbation dans les tâches requérant une organisation de l'information pourrait également expliquer pourquoi certains auteurs rapportent des troubles des fonctions visuo-spatiales dans la MP [15], alors que d'autres n'ont pu confirmer cette observation [16]. Il semble, en fait, que ces tâches de discrimination droite/gauche ou de rotation mentale échoueraient ou seraient réussies selon que le patient aurait à fournir lui-même une réponse, ou à choisir simplement la réponse parmi plusieurs alternatives [17]. Il est donc généralement admis $[17,18]$ que les déficits cognitifs dans la MP se retrouvent essentiellement dans les tâches qui requièrent un contrôle actif des processus cognitifs (contrôle interne du traitement de l'information). En revanche, les tâches qui ne requièrent qu'un traitement automatique de l'information, ou qui sont guidées par des indices externes (contrôle externe du traitement de l'information) sont toujours correctement exécutées.

La difficulté des parkinsoniens dans les tâches à contrôle interne résulterait d'une dysfonction des processus de l'attention [18]. Une hypothèse tirée de la psychologie cognitive suggère une mauvaise répartition des ressources de l'attention à travers les différentes opérations mentales qui doivent être activées au cours d'une tâche ou d'une situation non automatisée [19]. En d'autres termes, les troubles du contrôle interne seraient des troubles d'allocation des ressources de l'attention sur les composantes cognitives appropriées. Ce déficit d'allocation des ressources de l'attention dans la MP a pu être mesuré, notamment par l'exécution simultanée de tâches cognitives liées à la perception [20], ou des tâches de mémoire de travail qui requièrent la rétention d'une information pendant l'exécution d'une seconde tâche [21]. Les études faites chez l'homme et chez l'animal utilisant ce type de tâches montrent que les lésions des lobes frontaux ou des systèmes dopaminergiques mésocortical et nigro-strié, induisent des déficits comparables à ceux observés dans la MP [22].

\section{Contribution des systèmes dopaminergiques dans le SSCF de la MP}

La présence d'un SSCF dans la MP apparaît comme la conséquence d'une désafférentation du cortex frontal (figure 1). En effet, un trouble fonctionnel cortical prédomine chez ces patients dans les régions frontales, comme le révèlent la consommation réduite en oxygène ou en désoxyfluoroglucose [23], et l'apparition de troubles cognitifs analogues à ceux observés lors de lésions corticales frontales $[24,25]$. Le rôle de la dégénérescence dopaminergique nigro-striée, caractéristique de la MP, a été largement évoqué pour expliquer cette désafférentation frontale [25]. Ces études ont fait proposer que le SSCF résulterait d'une démodulation dopaminergique du striatum entraînant une perturbation de la circulation d'informations à l'intérieur de la boucle cortico-striato-pallido-thalamo-frontale (figure 1). Cette boucle peut être divisée en trois composantes fonctionnelles distinctes, sensori-motrice, associative (ou cognitive), et limbique, dont les fibres cheminent parallèlement (Tableau III). La démodulation de la 


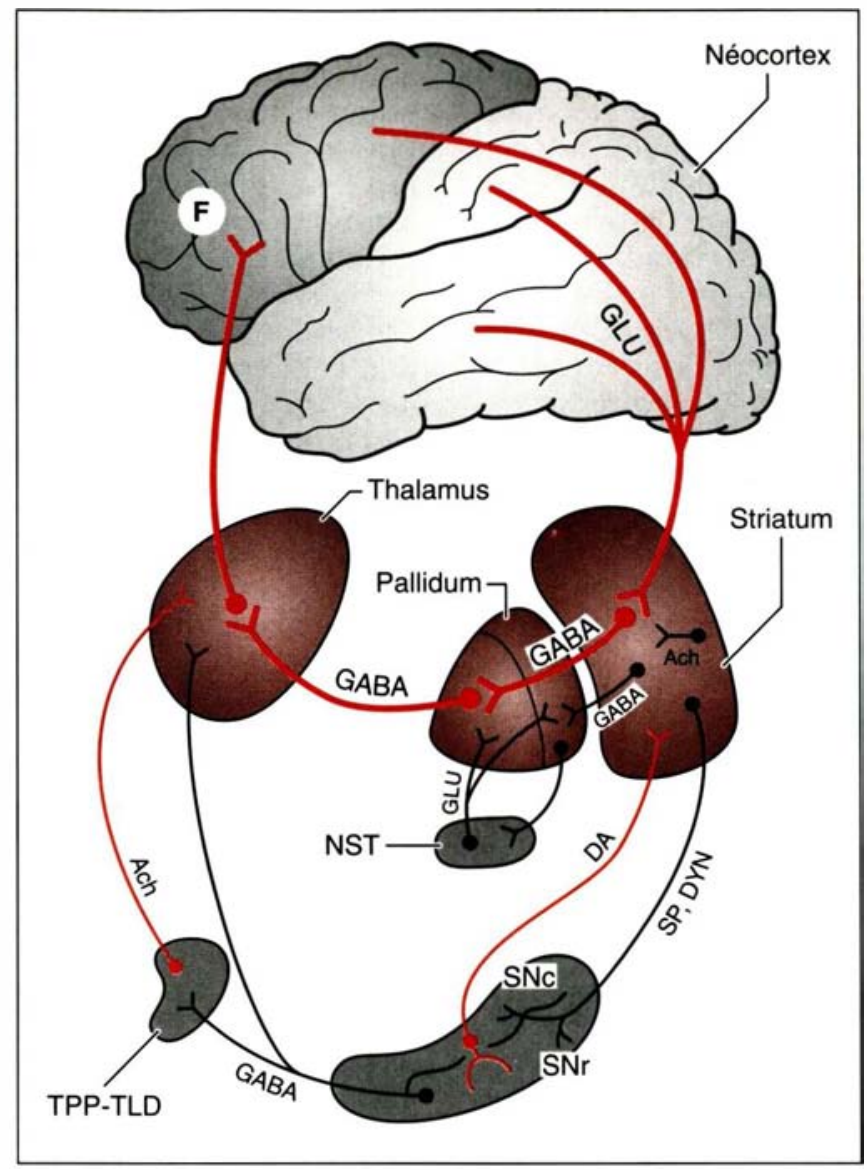

Figure 1. Organisation anatomique du cortex avec les noyaux gris centraux. L'ensemble du cortex cérébral se projette sur une voie striato-pallido-thalamique qui retourne au cortex frontal (trait rouge épais). En parallèle existent une boucle striatonigro-thalamique et une boucle pallido-sous-thalamique. L'ensemble est modulé par: des interneurones cholinergiques striataux; la voie dopaminergique nigrostriée; les voies sérotoninergiques ascendantes (qui n'aoparaissent pas sur le schéma); la voie cholinergique issue des noyaux tegmentaires pédonculo-pontin (TPP) et latéro-dorsal (TLD). Dans la $M P$, les trois boucles sont bien préservées mais l'ensemble des voies modulatrices sont atteintes à divers degrés. La voie dopaminergique nigro-striée semble être directement reliée au syndrome akinéto-rigide de la MP, alors que les fibres cholinergiques issues des noyaux TPP et TLD pourraient être plus déterminantes dans le SSCF de ces patients. Ach: acétylcholine; GABA: acide $\gamma$-aminobutyrique; GLU: glutamate ; DA: dopamine ; $S P$ : substance $P$; DYN: dynorphine; F: cortex frontal ; SNC: substance noire pars compacta ; SNr: substance noire pars reticulata ; NST: noyau sous-thalamique.

Tableau III

SUBDIVISION FONCTIONNELLE DE LA BOUCLE CORTICO-STRIATO-PALLIDO-THALAMO-FRONTALE

\begin{tabular}{|c|c|c|c|}
\hline & Système sensori-moteur & Système associatif & Système limbique \\
\hline $\begin{array}{c}\text { Cortex } \\
V\end{array}$ & $\begin{array}{l}\text { sensori-moteur } \\
\text { prémoteur }\end{array}$ & $\begin{array}{l}\text { associatif } \\
\text { - préfrontal } \\
\text { - pariétal } \\
\text { - temporal }\end{array}$ & $\begin{array}{l}\text { limbique } \\
\text { paralimbique }\end{array}$ \\
\hline Striatum & $\begin{array}{l}\text { 1/4 du noyau caudé (dorso-latéral) } \\
2 / 3 \text { du putamen (dorso-latéral) }\end{array}$ & $\begin{array}{l}3 / 4 \text { du noyau caudé } \\
1 / 3 \text { du putamen }\end{array}$ & striatum ventral \\
\hline $\begin{array}{l}\text { Pallidum } \\
\text { Substance noire }\end{array}$ & $\begin{array}{l}\text { 2/3 du pallidum (ventral) } \\
\text { SN (caudo-latérale) }\end{array}$ & $\begin{array}{l}1 / 3 \text { du pallidum (dorsal) } \\
\text { SN (rostro-médiane) }\end{array}$ & pallidum ventral \\
\hline Thalamus & $\begin{array}{l}\text { VA magnocellulaire (latéral) } \\
\text { VA parvocellulaire } \\
\text { (caudal, ventro-médian) } \\
\text { VL (oralis) } \\
\text { DM (denso- et parvocellulaire) }\end{array}$ & $\begin{array}{l}\text { VA magnocellulaire (médian) } \\
\text { VA parvocellulaire (rostral, dorsolatéral) } \\
\text { VL (medialis) } \\
\text { DM (magnocellulaire parafasciculaire) }\end{array}$ & DM \\
\hline Cortex & $\begin{array}{l}\text { moteur } \\
\text { prémoteur } \\
\text { - aire oculo-motrice } \\
\text { - aire motrice supplémentaire }\end{array}$ & $\begin{array}{l}\text { pré-frontal } \\
\text { - orbital } \\
\text { - dorso-latéral } \\
\text { (prémoteur) } \\
\text { (aire oculo-motrice) }\end{array}$ & $\begin{array}{l}\text { préfrontal } \\
\text { - orbital }\end{array}$ \\
\hline
\end{tabular}

VA: ventral antérieur; VL: ventro-latéral; DM: dorso-médian; SN: substance noire. 


\section{RÉFÉRENCES}

27. Stern Y, Langston JW. Intellectual changes in patients with MPTP-induced parkinsonism. Neurology 1985; 35 : 1506-9.

28. Cooper JA, Sagar HJ, Jordan N, Harvey NS, Sullivan EV. Cognitive impairment in early, untreated Parkinson's disease and its relationship to motor disability. Brain 1991 ; 114: 2095-122.

29. Pillon B, Dubois B, Cusimano G, Bonnet AM, Lhermitte F, Agid Y. Does cognitive impairment in Parkinson's disease result from non-dopaminergic lesions? J Neurol Neurosurg Psychiatr 1989; 52 : 201-6.

30. Rinne JO, Rummukainen J, Paljärui L, Rinne UK. Dementia in Parkinson's disease is related to neuronal loss in the medial substantia nigra. Ann Neurol 1989; 26: 4750.

31. Girotti F, Carella F, Pia Grassi M, el al. Motor and cognitive performances of parkinsonian patients in the "ON" and "OFF" phases of the disease. J Neurol Neurosurg Psychiatr 1986; 49: 657-60.

32. Gotham AM, Brown RG, Marsden CD Frontal cognitive function in patients with Parkinson's disease "on" and "off " levodopa. Brain 1988; 111 : 299-321.

33. Beardsley J, Puletti F. Personality (MMPI) and cognitive (WAIS) changes after levodopa treatment. Arch Neurol 1971 ; $25: 145-50$.

34. Loranger AW, Goodell H, Le JE, McDowell F. Levodopa treatment of Parkinson's syndrome improve intellectual functionning. Arch Gen Psychiatr 1972; 26: 163-8.

35. Portin R, Rinne UK. Neuropsychological responses of Parkinsonian patients to long-term levodopa treatment. In: Rinne UK, Klingler M, Stamm G, eds. Parkinson's disease: current progress, problems and management. Amsterdam: Elsevier-North-Holland, $1980: 271-304$.

36. Luciana M, Depue RA, Arbisi P, Leon A. Facilitation of working memory in humans by a D2 dopamine receptor agonist. J Cognit Neurosci $1992 ; 4: 58-68$.

37. Dubois B, Pillon B. Biochemical correlates of cognitive changes and dementia in Parkinson s disease. In: Huber S], Cummings JL, eds. Parkinson's disease: neurobehavioral aspects. Oxford: Oxford University Press, 1992: 178-98.

38. VanSpaendonck KPM, Berger HJC, Horstink MWI, Buytenhuijs EL, Cools ÅR. Impaired cognitive shifting in Parkinsonian patients on anticholinergic therapy. Neuropsychologia 1993; 31: 407-11.

39. Dubois B, Pillon B, Lhermitte F, Agid Y. Cholinergic deficiency and frontal dysfunction in Parkinson's disease. Ann Neurol 1990 ; 28: 117-21. boucle sensori-motrice serait à l'origine du syndrome akinéto-rigide de la MP [26]. On a postulé la même démodulation de la boucle associative pour expliquer le SSCF. L'élément le plus convaincant provient des études faites chez l'animal et chez l'homme exposés au 1-méthyl-4-phényl-1,2,3,6-té trahydropyridine (MPTP). Ce composé est un dérivé de la mépéridine de synthèse, une substance illicite utilisée dans les années 1970 en Californie. L'administration de MPTP produit une dégénérescence relativement sélective des cellules dopaminergiques de la substance noire, associée à un important syndrome akinéto-rigide et des troubles cognitifs rappelant ceux du SSCF [27]. L'hypothèse d'un rôle du système nigro-strié dans le SSCF est également confortée par les corrélations positives observées entre les niveaux d'akinésie de la MP, que l'on sait fortement reliés à ces lésions dopaminergiques, et le degré de perturbation cognitive chez ces patients [28]. En revanche, d'autres études [29] ont pu spécifier que parmi les signes moteurs de la MP, ceux qui ne sont pas associés à la dégénérescence nigro-striée, comme la dysarthrie ou les troubles de la marche, sont les mieux corrélés au SSCF. D’ailleurs, les tentatives de corrélations directes entre le degré de perte neuronale dans la substance noire et l'ampleur des déficits cognitifs dans la MP n'ont pas été concluantes quant à la contribution du système dopaminergique nigro-strié [7]. Il importe aussi de noter que dans la forme juvénile de la MP, caractérisée par une lésion nigro-striée isolée, on observe essentiellement un syndrome akinéto-rigide sans trouble cognitif, et cela pendant toute la durée d'évolution de la maladie [4]. Ces éléments soulignent donc l'importance de rechercher d'autres lésions sous-corticales dans la MP, qui pourraient contribuer de façon significative au SSCF.

Certains prétendent que les difficultés à trouver les relations entre lésions nigro-striées et déficits cognitifs dans la MP sont dues à la distribution topographique des lésions dans le striatum [30]. En effet, le déficit dopaminergique dans la MP prédomine dans le putamen. Or cette région correspond pour l'essentiel au territoire sensori-moteur du stria- tum et non au territoire cognitif ou associatif qui, lui, occupe surtout le noyau caudé (Tableau III). En se limitant essentiellement à la partie médiane de la substance noire, c'està-dire la portion qui se projette plus spécifiquement dans le noyau caudé, les corrélations entre dégénérescences dopaminergiques et détérioration cognitive deviennent statistiquement significatives [30]. Cependant, cet argument anatomique ne résiste pas à l'observation clinique qu'un rétablissement de la transmission dopaminergique dans la MP ne s'accompagne pas d'amélioration des fonctions cognitives, en dépit d'une amélioration des signes moteurs. Des travaux comparant les performances de patients parkinsoniens à des tests cognitifs spécifiques du SSCF au cours de périodes on (réponse thérapeutique maximale) et off (absence de réponse thérapeutique) ne montrent pas de différence significative [31, 32]. De faibles améliorations cognitives avaient été rapportées avec un traitement par la L-dopa chez des patients dont l'évaluation du rendement intellectuel était réalisée à l'aide de mesures globales [33, 34] ; mais ces résultats n'ont pu être retrouvés par la suite, lors de mesures plus spécifiques du SSCF. Des études longitudinales [35] ont même montré que l'amélioration cognitive parfois observée au moment du début d'un traitement par la L-dopa était rapidement suivie d'un retour aux faibles performances de base. Les résultats positifs initiaux ont donc été attribués à un effet stimulant général de la dopamine, ou à un effet non spécifique lié au mieux-être chez les malades traités.

Les nouveaux modèles de la psychologie cognitive ont permis une étude plus spécifique des troubles cognitifs de la MP qui répondent aux traitements dopaminergiques. Des résultats récents [20] montrent que les traitements dopaminergiques affecteraient spécifiquement la performance des parkinsoniens dans les tâches qui font appel à une allocation adéquate des ressources de l'attention, c'est-à-dire les tâches qui demandent un traitement simultané de plusieurs informations. Cela rejoint les résultats obtenus chez le sujet sain [36] et chez l'animal [22], traités par divers agents agonistes et antagonistes de la 
dopamine. De tels résultats portent toutefois à controverse car, si l'on suppose, comme d'autres [2(), 21], que le SSCF de la MP résulte d'un trouble central d'allocation des ressources de l'attention et que, par ailleurs, la dopamine joue un rôle primordial dans ce déficit, pourquoi les traitements dopaminergiques ne sont-ils pas efficaces pour éliminer l'ensemble du SSCF de la MP? D'autres systèmes neurochimiques jouent certainement un rôle primordial.

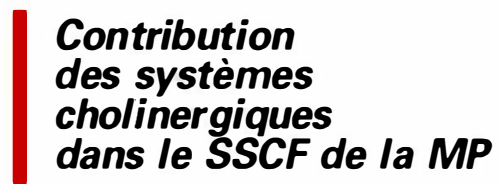

Des études post-mortem ont montré une perte massive des cellules cholinergiques dans le cerveau de patients parkinsoniens [7-9, 37]. Des régions diffuses du cortex sont affectées, comme le révèle la réduction des concentrations de choline-acétyltransférase et des récepteurs muscariniques dans les lobes frontaux, pariétaux et occipitaux, et dans les hippocampes [37], laissant suggérer que cette déplétion pourrait contribuer aux troubles cognitifs de ces patients. Malheureusement, ces études ont souvent considéré la coexistence d'une démence comme critère de détérioration cognitive, sans distinction véritable entre une démence de type Alzheimer ou un SSC.F isolé. Le degré d'atteinte des fibres innominato-corticales et septohippocampiques se situe à environ 7()-80 \% chez ces parkinsoniens avec démence, alors qu'il se situe à environ $20 \%$ chez ceux qui ne présentent pas de démence associée [9, 37]. On voit mal, cependant, comment ces lésions cholinergiques qui affectent l'ensemble du cortex pourraient être spécifiquement impliquées dans le tableau clinique caractéristique du SSCF.

Plusieurs études montrent que des parkinsoniens sans trouble cognitif, auxquels on administre un traitement anticholinergique, développent au moment du traitement un SSC.F caractéristique [38-41]. La sélectivité des déficits cognitifs et les faibles doses utilisées dans ces études permettent d'écarter l'hypothèse de l'induction d'un syndrome confu- sionnel ou d'une démence de type Alzheimer parfois observés avec de fortes doses d'anticholinergiques [42]. Par ailleurs, l'administration aiguë de scopolamine à une dose infraliminaire, c'est-à-dire une dose qui reste sans effet chez des sujets sains, produit chez des parkinsoniens un SSCF pendant toute la durée d'action du médicament [40, 41]. On connaissait déjà les effets délétères des anticholinergiques administrés à plus fortes doses chez le sujet sain [42]. C.es effets se rapprochent des déficits observés dans les démences de type Alzheimer, ce qui suggère que l'effet des anticholinergiques sur les fonctions cognitives peut être relié à leur action inhibitrice sur les systèmes cholinergiques innominato-cortical et septo-hippocampique. Toutefois, l'apparition spécifique d'un SSCF dans la MP pour des doses infraliminaires de scopolamine suppose un mécanisme différent. Il est possible que ces faibles doses n'affectent que les systèmes cholinergiques les plus atteints dans la MP et que, comme pour les sujets sains, les systèmes mieux préservés ne soient pas affectés par ces faibles doses.

Parmi les systèmes cholinergiques les plus touchés dans la MP, les noyaux tegmentaires pédonculo-pontin (NTPP) et latéro-dorsal (NTLD) pourraient être plus particulièrement critiques pour expliquer le SSCF (figure 1). Une perte cellulaire importante a été rapportée dans ces noyaux $[43,44]$ et cette perte cholinergique est corrélée à la perte dopaminergique observée dans la substance noire [44]. Le NTPP et le NTLD sont connus pour leurs projections essentiellement sous-corticales, notamment au noyau médio-dorsal du thalamus, structure impliquée dans la boucle cortico-striato-pallidothalamo-frontale (figure 1). On peut donc proposer l'hypothèse que cette démodulation cholinergique, seule ou en association avec la démodulation dopaminergique nigro-striée, pourrait contribuer à l'apparition du SSCF. Un des arguments en faveur de cette hypothèse est que dans une maladie comme la paralysie supranucléaire progressive, où le SSCF est encore plus sévère que dans la MP, le NTPP et le NTLD sont les seules structures cholinergiques touchées
[45]. Cette hypothèse cholinergique du SSC.F reste encore à confirmer. Ainsi, un agoniste cholinergique tel que la physostigmine s'est avéré efficace pour améliorer les performances cognitives chez certains malades atteints de paralysie supranucléaire progressive [46], mais est resté sans effet chez d'autres [47]. Le rôle prépondérant des NTPP/NTLD pourrait être plus spécifiquement démontré à l'aide d'un modèle animal, dans lequel des lésions limitées à ces noyaux reproduiraient des déficits comportementaux analogues à ceux observés lors d'une lésion du cortex préfrontal. La réversibilité de ces déficits "frontaux" après administration d'agonistes cholinergiques directs permettrait de confirmer avec une plus grande certitude le rôle des systèmes cholinergiques issus du complexe NTPP/NTLD.

\section{Contribution des autres systèmes neurochimiquement définis}

Les lésions noradrénergiques de la MP concernent essentiellement le locus copruleus. Cette réduction noradrénergique qui touche l'ensemble du cortex et des structures limbiques est présente surtout chez les malades atteints d'une démence sévère [37]. Cette déplétion noradrénergique s'accompagne d'une augmentation du nombre des récepteurs $\beta 1$ (postsynaptiques) et d'une réduction des récepteurs $\alpha 2$ (pré- et post-synaptiques), surtout localisées dans le cortex frontal. Il semble que ces lésions noradrénergiques aient un rôle à jouer dans les déficits de l'attention des parkinsoniens. Les dosages dans le liquide céphalorachidien montrent en effet que les concentrations du métabolite extracellulaire de la noradrénaline, le 3-méthoxy-4hydroxyphénéthylèneglycol (MHPG) sont corrélées positivement aux performances lors d'un test d'attention sélective (continuous performance task) [48]. De plus, des résultats que nous avons obtenus récemment montrent que l'administration d'un agoniste $\alpha$ peut améliorer les performances des parkinsoniens à plusieurs tests cognitifs qui impliquent une attention sélective [49]. Il semble donc qu'une partie des troubles cognitifs 


\section{RÉFÉRENCES}

40. Bédard MA, Masson $H$, Perreault $H$, Gaudreau S. Specific memory deficits related to lesions of the cholinergic systems in Parkinson's disease. In: Amos D. Korczyn, eds. Dementia in Parkinson's disease. Bologna: Monduzzi Editore, 1994: 213-6.

41. Dubois B, Danzé F, Pillon B, Cusimano G, Lhermitte F, Agid Y. Cholinergic-dependent cognitive deficits in Parkinson's disease. Ann Neurol 1987; 22: 26-30.

42. Drachman DA, Leavitt J. Human memory and the cholinergic system. Arch Neurol $1974 ; 30: 113-21$.

43. Hirsch E, Graybiel AM, Duyckaerts C, Javoy-Agid F. Neuronal loss in the pedunculopontine tegmental nucleus in Parkinson's disease and in progressive supranuclear palsy. Proc Natl Acad Sai USA 1987; 84: 5976-80.

44. Zweig RM, Jankel WR, Hedreen JC, Mayeux R, Price DL. The pedunculopontine nucleus in Parkinson's disease. Ann Neurol $1989 ; 26: 41-6$.

45. Malessa S, Hirsch E, Cervera P, JavoyAgid F, Duyckaerts C, Hauw II, Agid Y. Progressive supranuclear palsy: loss of cholineacetyltransferase-like immunoreactive neurons in the pontine reticular formation. Neurology $1991 ; 41$ : 1593-7.

46. Litvan I, Gomez C, Atack J, Gillespie M, Kask AM, Mouradian M, Chase T. Physostigmine treatment of progressive supranuclear palsy. Ann Neurol 1989;26: 404-7.

47. Kertzman C, Robinson DL, Litvan I. Effects of physostigmine on spatial attention in patients with progressive supranuclear palsy. Arch Neurol 1990; 47: 1346-50.

48. Stern Y, Mayeux R, Coté L. Reaction time and vigilance in Parkinson's disease: possible role of norepinephrine metabolism. Arch Neurol 1984; 41 : 1086-9.

49. Bédard MA, El Massioui F, Nandrino JL, Dubois $B$. Covert orienting of attention in Parkinson's disease. Neurology 1992; 42 : 170 .

50. Mayeux R, Stern Y, Williams JBW, Frantz A, Cote L, Dyrenfurth I. Clinical and biochemical features of depression in Parkinson's disease. Am J Psychiatr 1985; 143: 756-9.

51. Cooper JA, Sagar HJ, Doherty SM, Jordan N, Tidswell P, Sullivan V. Different effect of dopaminergic and anticholinergic therapies on cognitive and motor function in Parkinson's disease. Brain 1992; 115 : des parkinsoniens puisse s'expliquer par l'atteinte des systèmes noradrénergiques ascendants. Certains signes cliniques et pharmacologiques suggèrent aussi un rôle de ces systèmes dans le syndrome dépressif souvent observé dans la MP [50].

La même analyse peut être faite également à propos des lésions du système sérotoninergique ascendant issu des noyaux du raphé. Ces neurones sont partiellement atteints dans la MP et les dosages dans le liquide céphalorachidien ont montré des corrélations entre les faibles concentrations du métabolite de la sérotonine, l'acide 5-hydroxy-indole-acétique (5-HIAA), et la présence d'une dépression [37]. Dans le cortex cérébral, les concentrations de sérotonine ont été trouvées réduites chez les parkinsoniens avec ou sans démence [37], et cela plus particulièrement dans le cortex frontal et les hippocampes cérébraux. Toutefois, le nombre de récepteurs sérotoninergiques semble normal chez ces patients, et on dispose de peu d'éléments en faveur d'un rôle spécifique de ces lésions dans les troubles cognitifs de la MP.

\section{Conclusion}

Les lésions neurochimiques de la MP sont nombreuses et incluent, d'une part, des structures qui projettent directement au cortex, comme l'aire tegmentaire ventrale, le locus coeruleus, les noyaux du raphé et le noyau basal de Meynert et, d'autre part, des structures dont les projections sont sous-corticales, comme la substance noire et le complexe NTPP/NTLD. Ces systèmes n'agissent pas séparément mais plutôt de façon parallèle et/ou interactive sur l'ensemble du tableau parkinsonien. On sait, par exemple, que les fibres dopaminergiques de l'aire tegmentaire ventrale reçoivent des afférences cholinergiques issues des NTPP/NTLD. On ne peut donc attribuer une sémiologie aussi complexe que le SSCF au dysfonctionnement d'un seul système neurochimique isolé. De la même façon, il n'est pas possible de rapporter l'ensemble des lésions neurochimiques de la MP à un déficit cognitif central qui expliquerait le SSCF. Par conséquent, les rôles respectifs de chacune des lésions neurochimiques de la MP restent inconnus, bien que nous sachions que certaines d'entre elles jouent un rôle primordial dans la sémiologie motrice et que d'autres sont plus déterminantes dans les déficits cognitifs [37]. Ainsi, l'atteinte dopaminergique de la substance noire serait étroitement reliée au syndrome akinéto-rigide de la MP, alors que l'atteinte des fibres cholinergiques du noyau basal de Meynert est fréquemment associée à la présence d'une démence de type Alzheimer [7-9, 37]. Par ailleurs, l'atteinte du complexe NTPP/NTLD, seule ou associée à l'atteinte de la substance noire (pars compacta), pourrait être impliquée dans le SSCF de la MP. Toutefois, les rôles respectifs de ces deux derniers systèmes neurochimiques demandent encore à être précisés. Le développement d'un modèle animal du SSCF permettrait certainement d'éclaicir cette question. Chez l'homme, des résultats récents ont montré que les traitements dopaminergiques de la MP (Ldopa et bromocriptine) n'affectent pas les mêmes déficits cognitifs que ceux touchés par les traitements anticholinergiques (amantadine et trihexyphénidyl), même si, dans les deux cas, il s'agit de fonctions faisant partie intégrante du SSCF [51]. D'autres déficits cognitifs notés chez ces patients pourraient être indépendants des traitements dopaminergiques ou cholinergiques. Ce type d'études, utilisant les nouvelles approches tirées de la neuropsychologie fonctionnelle (ou cognitive) et de la neuropharmacologie clinique, outre qu'il offre une perspective de traitements mieux adaptés, permet d'envisager une compréhension plus satisfaisante des troubles cognitifs propres à la MP

\section{TIRÉS À PART}

M.A. Bédard. 


\section{Summary}

Neurochemistry of cognitive functions in Parkinson's disease

Cognitive functions in Parkinson's disease (PD) have received attention over the last two decades. Knowledge about the nature, the prevalence and the pathophysiology of cognitive dysfunctions in PD has significantly progressed. Alzheimer's disease seems to be more frequent in $\mathrm{PD}$ than in the general population. Diffuse Lewy Body Disease may also be another cause of dementia in PD. In addition, most patients with PD show a specific picture of cognitive deficits called the subcortico-frontal syndrome (SCFS) that is characterized by a frontal lobe-like syndrome. SCFS in PD seems to result from a dysfunction of the subcortical projections to the frontal cortex. The complete biochemistry of these projections and the cognitive functions that they underly remain misunderstood. Until now, most studies have focused on the well known dopaminergic degenerations of PD. However, the cholinergic systems - and more precisely the pedunculopontine and laterodorsal tegmental nuclei - with their interactions with dopaminergic lesions, could be more fruitfull in the understanding of the neurochemistry of the SCFS.

\section{* GLOSSAIRE *}

Akinésie : trouble caractérisé par une diminution ou une disparition des mouvements spontanés et automatiques et une lenteur du mouvement volontaire, en l'absence de lésion de la voie motrice principale.

Bradykinésie : lenteur des mouvements automatiques ou volontaires en l'absence de troubles de la vigilance observés dans les syndromes extrapyramidaux et, en particulier, la maladie de Parkinson.

Dysarthrie : trouble de l'élocution lié à une atteinte neurologique des systèmes moteurs participant à l'expression de la parole, du système cérebelleux et strié (au cours des syndromes parkinsoniens).

Persévération : terme général indiquant le maintien d'une attitude, la répétition d'un geste ou de mots.

Rigidité pallidale : hypertonie intense liée à une lésion du pallidum, essentiellement dans sa partie interne, au cours de la maladie de Parkinson.

Tremblement de repos : tremblement apparaissant au repos et disparaissant au cours du mouvement, observé dans les syndromes parkinsoniens. 